\title{
Association between postoperative hyponatremia and renal prognosis in major urologic surgery
}

\author{
Sehoon Park ${ }^{1,4}$, Jung Nam An², Jung Pyo Lee ${ }^{2,3}$, Yun Kyu Oh', ${ }^{2,3}$ Dong Ki Kim ${ }^{3,4}$, \\ Kwon Wook Joo ${ }^{3,4}$, Yon Su Kim ${ }^{1,4}$ and Chun Soo Lim ${ }^{2,3}$ \\ ${ }^{1}$ Department of Biomedical Sciences, Seoul National University College of Medicine, Seoul, Korea \\ ${ }^{2}$ Department of Internal Medicine, Seoul National University Boramae Medical Center, Seoul, Korea \\ ${ }^{3}$ Department of Internal Medicine, Seoul National University College of Medicine, Seoul, Korea \\ ${ }^{4}$ Department of Internal Medicine, Seoul National University Hospital, Seoul, Korea
}

Correspondence to: Chun Soo Lim, email: cslimjy@snu.ac.kr

Keywords: hyponatremia, urology, acute kidney injury, end stage renal disease, surgery

Received: May 30, 2017 Accepted: August 04, $2017 \quad$ Published: August 18, 2017

Copyright: Park et al. This is an open-access article distributed under the terms of the Creative Commons Attribution License 3.0 (CC BY 3.0), which permits unrestricted use, distribution, and reproduction in any medium, provided the original author and source are credited.

\section{ABSTRACT}

Recent evidence for the clinical significance of postoperative hyponatremia after urologic surgeries remains scarce. We examined the incidence, risk factors, and outcomes of electrolyte imbalance in urologic surgery. Patients with newly developed hyponatremia, defined as a sodium level lower than $135 \mathrm{mEq} / \mathrm{L}$ within 7 days after surgery, were included in our study group. The primary outcome was progression to end-stage renal disease (ESRD). Secondary outcome was composition of progression to ESRD and creatinine doubling/eGFR halving from baseline. A survival analysis with a multivariable Cox proportional hazard model was performed. We included 9,206 cases of bladder, prostate, ureter, and kidney surgery. Incidence of new-onset postoperative hyponatremia was $15.4 \%(1,414 / 9,206)$. Postoperative hyponatremia mostly developed in patients with high-risk perioperative characteristics. The development of postoperative hyponatremia was independently associated with progression to ESRD (adjusted HR 1.343, 95\% CI 1.082-1.680, $P=0.007$ ). The secondary outcome was also related to the electrolyte imbalance in prostate (adjusted HR 1.729, 95\% CI 1.145-2.612, $P=0.009$ ) and kidney (adjusted HR $1.339,95 \%$ CI 1.099-1.632, $P=0.004$ ) surgery. Postoperative hyponatremia in urologic surgery was a common electrolyte imbalance in patients with high-risk perioperative status, and associated with worse renal prognosis.

\section{INTRODUCTION}

Hyponatremia is the most common electrolyte imbalance in hospitalized patients $[1,2]$. Previous studies have shown a close relationship between in-hospital hyponatremia and adverse clinical outcomes [2-6]. As surgery was found to be one of the main causes of inhospital hyponatremia, some have investigated the clinical significance of postoperative hyponatremia in several fields, including neurosurgery, orthopedic operations, and urologic surgeries [3-5, 7-9]. However, recent studies considering electrolyte imbalance in urologic surgery have focused on the classic concept of transurethral-resection of prostate (TUR-P) syndrome [7,8], although advances in technique have significantly decreased its incidence [10]. Hence, incidence and risk factors for postoperative hyponatremia in recent major urologic surgery need further investigation.

In addition, as the urinary system is directly manipulated and many disease entities are closely related to kidney function in urologic practice, few previous studies investigated AKI after urologic operation and demonstrated its significant impact on patient prognosis [11-13]. Considering that postoperative hyponatremia and AKI are both common in patients with perioperative risk factors $[3,5,6,9,14,15]$, one could assume that postoperative hyponatremia would also be related to worse postoperative prognosis [9]. However, whether 
postoperative hyponatremia is a risk factor for worse renal outcomes, independent from stages of kidney injury or other clinical factors, has not been determined.

In this study, we aimed to assess whether newonset postoperative hyponatremia is associated with longterm renal prognosis in the urology field. We analyzed patients who underwent four major categories of recent urologic surgeries: bladder, prostate, ureter, and kidney, and investigated the prevalence, risk factors, and clinical outcomes of new-onset postoperative hyponatremia.

\section{RESULTS}

\section{Study population and incidence of postoperative hyponatremia}

A total of 26,292 cases of urologic surgery were screened for study enrollment (Figure 1). Many patients were excluded due to non-available laboratory results of serum $\mathrm{Na}$ within 7 days after operation $(n=12,407)$, most of which were minor procedures such as vasectomy, circumcision, and wound repair. The two other main causes of exclusion were cases which could not be categorized into bladder, prostate, ureter, and kidney operations ( $n=$ $1,326)$ and those lost to follow up within 1-month postsurgery $(n=2,622)$. After exclusion, 9,206 cases of urologic surgery were included in our study. We identified major decrements in serum $\mathrm{Na}$ levels from baseline to the postoperative period in our study population (Figure 2). The incidence of new-onset postoperative hyponatremia was $15.4 \%(n=1,414)$. Patients with hyponatremia development were included in the study group, while the others were considered as the control group. Most of the study group had mild to moderate hyponatremia, sodium range of 125-134 $\mathrm{mEq} / \mathrm{L}(n=1,312)$, and 102 patients had severe post-operative hyponatremia $(\mathrm{Na}<125 \mathrm{mEq} / \mathrm{mL})$.

\section{Characteristics of patients with postoperative hyponatremia}

Regarding preoperative patient characteristics (Table $1)$, the study group were more commonly female $(P<$ $0.001)$, had a lower BMI $(P<0.001)$, and lower baseline hemoglobin $(P<0.001)$ and serum Na levels $(P<0.001)$

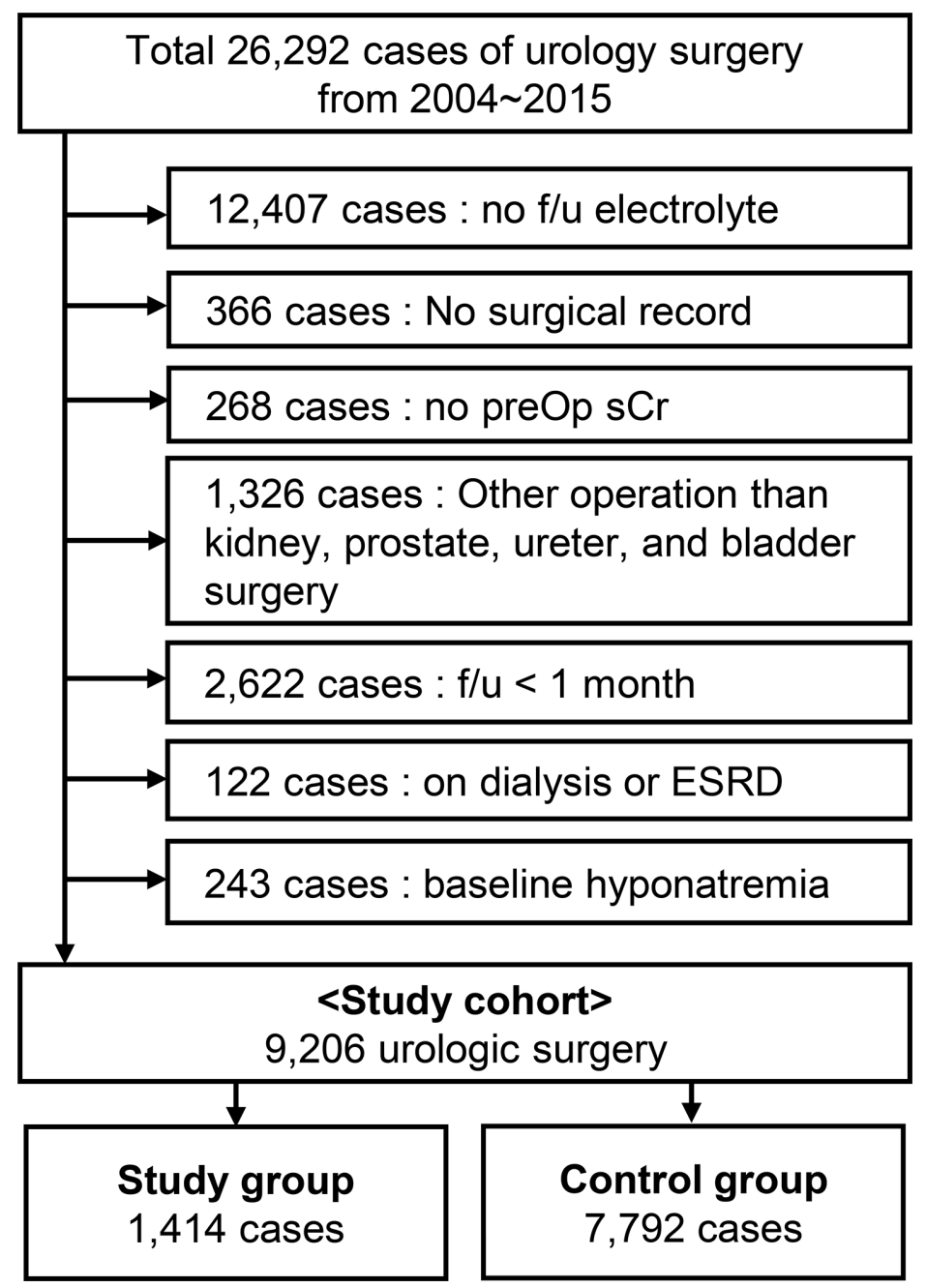

Figure 1: Flow diagram of the study population. 
than the control group. Comorbidities, such as hypertension $(P<0.001)$, diabetes mellitus $(P=0.023)$, and cancer $(P<$ $0.001)$, were more common in the study group. In contrast, baseline serum creatinine $(P=0.229)$ and eGFR $(P=$ 0.164 ) were not significantly different between groups. No significant difference in preoperative medication use was identified between the study and the control groups.

With regard to operation type (Table 2), the incidence of postoperative hyponatremia was significantly different according to the surgery category. Among the four operation categories, hyponatremia was most frequently (27\%) observed in patients of kidney operation, while incidences of the electrolyte imbalance were similar in bladder $(12.5 \%)$, prostate $(12.3 \%)$, and ureter $(10.9 \%)$ operations.

In addition, perioperative characteristics (Table 3) revealed that new-onset postoperative hyponatremia was associated with longer surgical/anesthesia time $(P<0.001)$ and higher preoperative risk stratification scores in terms of ASA classification $(P<0.002)$. The study group more frequently received common volume expanders including normal saline $(P<0.001)$, Hartman solution $(P=0.010)$, and hydroxyethyl starch $(P<0.001)$, and were infused with larger volumes of Hartman solution when used $(P<0.001)$. Increased RBC transfusion amounts were also observed in patients with postoperative hyponatremia $(P<0.001)$.

Lastly, postoperative characteristics were also significantly different between the study and control groups (Table 4). Postoperative hyponatremia was significantly related to acute kidney injury $(P<0.001)$. The study group also had a longer hospital stay $(P<$ $0.001)$, but the frequency of postoperative ICU admission was not significantly different between groups $(P=0.114)$.

\section{Risk factors for postoperative hyponatremia}

Outcomes related to risk factors for new-onset postoperative hyponatremia are summarized in Table 5. Multivariable analysis revealed age (adjusted OR 1.013, 95\% CI 1.007-1.018, $P<0.001$ ), female sex (adjusted OR 1.216, 95\% CI 1.045-1.525, $P=0.011$ ), history of diabetes mellitus (adjusted OR 1.317, 95\% CI 1.116-1.554, $P=0.001$ ), and cancer (adjusted OR 1.248, 95\% CI 1.067-1.459, $P=0.006$ ) were independent risk factors for hyponatremia development. Regarding laboratory results, baseline serum $\mathrm{Na}$ (adjusted

\section{Distribution of $\mathrm{Na}$}

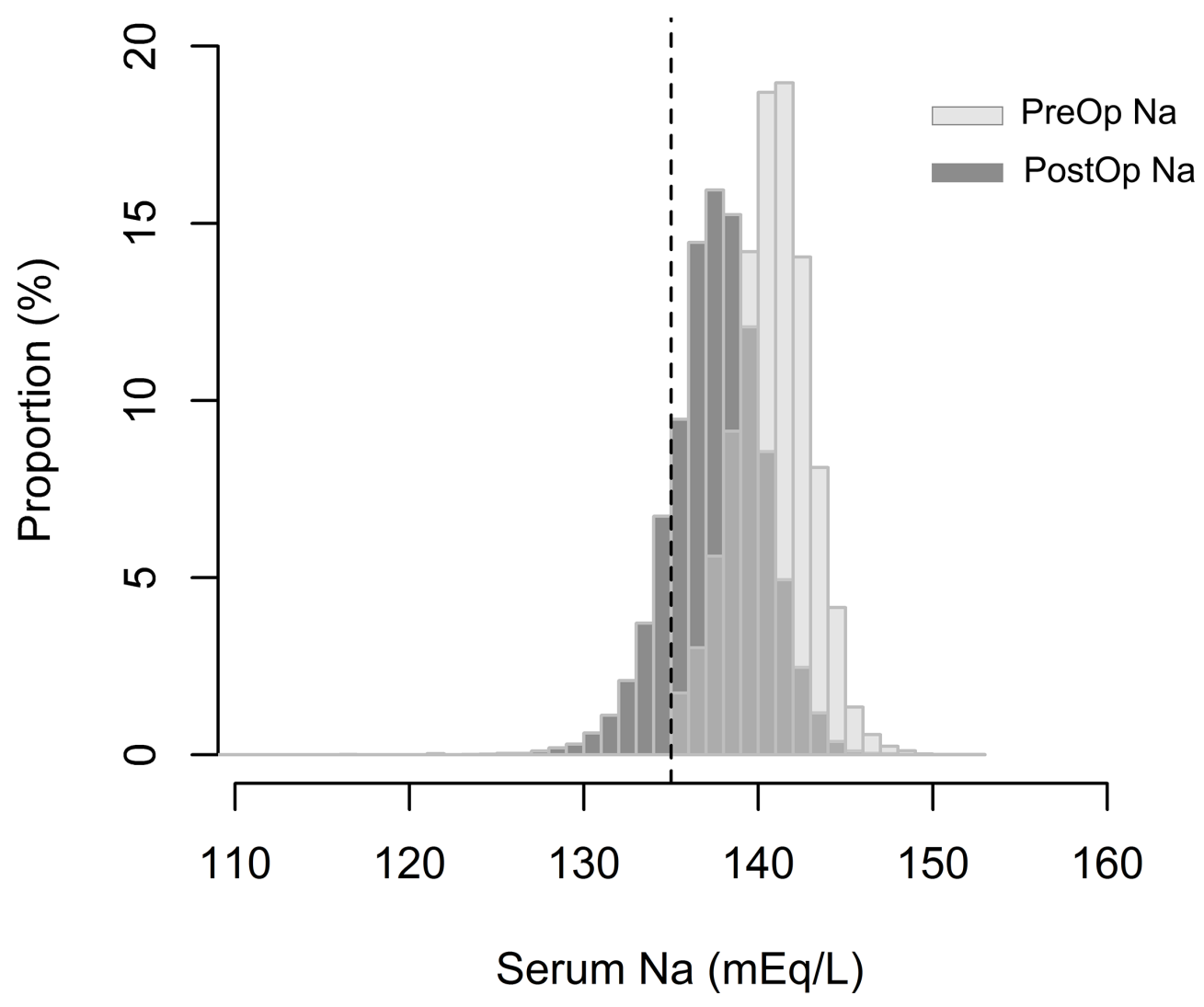

Figure 2: Distribution of lowest serum Na levels in the preoperative and postoperative periods in the study population. The X-axis shows the serum $\mathrm{Na}$ level and Y-axis shows the proportion of patients identified with the serum Na level. Light grey bars indicate the distribution of preoperative $\mathrm{Na}(\mathrm{PreOp} \mathrm{Na})$, and dark grey bars indicate the distribution of postoperative $\mathrm{Na}$ (PostOp $\mathrm{Na}$ ) values of the study population. Dotted vertical line shows a serum $\mathrm{Na}$ level of $135 \mathrm{mEq} / \mathrm{L}$, which was the criterion for hyponatremia in the study. 
Table 1: Baseline characteristics of study patients

\begin{tabular}{|c|c|c|c|}
\hline & $\begin{array}{c}\text { New-onset } \\
\text { hyponatremia }(+) \\
(N=1,414)\end{array}$ & $\begin{array}{c}\text { New-onset } \\
\text { hyponatremia }(-) \\
(N=7,792)\end{array}$ & $P$ value \\
\hline Age (years) & $65(54-72)$ & $65(54-71)$ & 0.065 \\
\hline Sex (Female) & $375(26.5)$ & $1657(21.3)$ & $<0.001$ \\
\hline Body mass index $\left(\mathrm{kg} / \mathrm{m}^{2}\right)$ & $24.0(22.1-26.0)$ & $24.3(22.3-26.4)$ & $<0.001$ \\
\hline \multicolumn{4}{|l|}{ Baseline laboratory results } \\
\hline $\mathrm{sCr}(\mathrm{mg} / \mathrm{dL})$ & $0.92(0.77-1.1)$ & $0.92(0.80-1.08)$ & 0.229 \\
\hline $\mathrm{eGFR}\left(\mathrm{mL} / \mathrm{min} / 1.73 \mathrm{~m}^{2}\right)$ & $68.7(52.9-90.5)$ & $67.6(54.1-86.5)$ & 0.164 \\
\hline Hemoglobin (g/dL) & $13.2(11.6-14.4)$ & $13.6(12.3-14.7)$ & $<0.001$ \\
\hline Sodium $(\mathrm{mEq} / \mathrm{L})$ & $140(138-141)$ & $141(139-142)$ & $<0.001$ \\
\hline \multicolumn{4}{|l|}{ Underlying comorbidities } \\
\hline Cancer & $1111(78.6)$ & $5691(73.0)$ & $<0.001$ \\
\hline Hypertension & $551(39.0)$ & $2630(33.8)$ & $<0.001$ \\
\hline Diabetes mellitus & $298(21.1)$ & $1441(18.5)$ & 0.023 \\
\hline Chronic kidney disease & $502(35.5)$ & $2730(35.0)$ & 0.735 \\
\hline Liver cirrhosis & $67(4.7)$ & $312(4.0)$ & 0.201 \\
\hline Heart failure & $104(7.4)$ & $491(6.3)$ & 0.138 \\
\hline \multicolumn{4}{|l|}{ Preoperative use of } \\
\hline ACE I/ARBs & $165(11.7)$ & $853(10.9)$ & 0.426 \\
\hline Diuretics & $150(10.6)$ & $723(9.3)$ & 0.116 \\
\hline SSRI/SNRIs & $14(1.0)$ & $97(1.2)$ & 0.419 \\
\hline NSAIDs & $273(19.3)$ & $1649(21.2)$ & 0.114 \\
\hline Antipsychotics & $11(0.8)$ & $43(0.6)$ & 0.306 \\
\hline Benzodiazepines & $42(3.0)$ & $242(3.1)$ & 0.786 \\
\hline Antiepileptic agents & $46(3.3)$ & $211(2.7)$ & 0.252 \\
\hline Proton pump inhibitor & $95(6.7)$ & $578(7.4)$ & 0.353 \\
\hline Thyroidal hormones & $19(1.3)$ & $92(1.2)$ & 0.605 \\
\hline Anti-thyroidal medications & $1(0.1)$ & $13(0.2)$ & 0.393 \\
\hline Oral steroids & $46(3.3)$ & $282(3.6)$ & 0.495 \\
\hline
\end{tabular}

$\mathrm{sCr}$, serum creatinine, eGFR, estimated glomerular filtration rate, ACE I, angiotensin converting enzyme inhibitor, ARB, angiotensin receptor blocker, SSRI, selective-serotonin reuptake inhibitor, SNRI, serotonin norepinephrine reuptake inhibitor, NSAID, non-steroidal anti-inflammatory drug

Values were presented as $n(\%)$ for categorical variables, and median ( $25 \%$ to $75 \%$ interquartile) for continuous variables (all data in the table showed non-normal distribution).

OR $0.829,95 \%$ CI $0.803-0.856, P<0.001)$, and anemia at baseline (adjusted OR 1.311, 95\% CI 1.139-1.509, $P<0.001$ ) were significantly associated with new-onset postoperative hyponatremia. In addition, kidney operation (adjusted OR $2.765,95 \%$ CI 2.388-3.202, $P<0.001$ ), longer surgical time (adjusted OR 1.109, 95\% CI 1.063-1.157, $P<0.001$ ), ASA class III or higher (adjusted OR 1.621, 95\% CI 1.372-1.915, $P<0.001$ ), and intraoperative use of normal saline (adjusted OR $1.236,95 \%$ CI $1.079-1.416, P=0.002$ ) or Hartman solution (adjusted OR 1.319, 95\% CI 1.107-1.573, $P=0.002$ ) were independently related to the presence of postoperative hyponatremia. Furthermore, postoperative AKI was strongly associated with the development of electrolyte imbalance and the risk showed increasing tendency in higher AKI stages.

\section{Risk of progression to ESRD in patients with postoperative hyponatremia}

Six-hundred and four cases of progression to ESRD were documented, $122(8.6 \%)$ in the study group, and $482(6.2 \%)$ in the control group. The median duration to progression to ESRD was $0.5(0.2-2.0)$ years in the study group, which was shorter than the median duration in the control group (1.1 [0.3-3.0] years). When we plotted the risk 
Table 2: Categories of operations and incidence of hyponatremia

\begin{tabular}{|c|c|c|c|c|}
\hline Operation category & $\begin{array}{l}\text { ancidence of } \\
\text { hyponatremia }\end{array}$ & $\begin{array}{c}\text { New-onset } \\
\text { hyponatremia }(+) \\
(N=1,414)\end{array}$ & $\begin{array}{c}\text { New-onset } \\
\text { hyponatremia }(-) \\
(N=7,792)\end{array}$ & $P$ values \\
\hline Bladder & $11.1 \%$ & $201(14.2)$ & $1,613(20.7)$ & $<0.001$ \\
\hline TUR-B & $8.1 \%$ & $86(6.1)$ & $980(12.6)$ & \\
\hline Cystolithotripsy & $4.8 \%$ & $3(0.2)$ & $60(0.8)$ & \\
\hline Cystectomy & $18.9 \%$ & $101(7.1)$ & $433(5.6)$ & \\
\hline Cystocele repair & $1.8 \%$ & $1(0.1)$ & $60(0.8)$ & \\
\hline Transurethral coagulation & $20 \%$ & $3(0.2)$ & $12(0.2)$ & \\
\hline Cystostomy & $0 \%$ & $0(0.0)$ & $4(0.1)$ & \\
\hline Other cystoscopy & $6.1 \%$ & $1(0.1)$ & $13(0.2)$ & \\
\hline miscellaneous & $10.5 \%$ & $6(0.4)$ & $51(0.7)$ & \\
\hline Prostate & $10.9 \%$ & $323(22.8)$ & $2,634(33.8)$ & $<0.001$ \\
\hline TUR-P, HoLEP etc. & $8.8 \%$ & $52(3.7)$ & $540(6.9)$ & \\
\hline Radical prostatectomy & $11.5 \%$ & $271(19.2)$ & $2,084(26.7)$ & \\
\hline miscellaneous & $0 \%$ & $0(0.0)$ & $10(0.1)$ & \\
\hline Ureter & $9.8 \%$ & $59(4.2)$ & $540(6.9)$ & $<0.001$ \\
\hline Stone removal & $9.1 \%$ & $30(2.1)$ & $301(3.9)$ & \\
\hline Ureterectomy & $22.2 \%$ & $4(0.3)$ & $14(0.2)$ & \\
\hline Ureterotomy & $7.8 \%$ & $4(0.3)$ & $47(0.6)$ & \\
\hline Ureteral stent insertion/removal & $13 \%$ & $13(0.9)$ & $87(1.1)$ & \\
\hline Other ureteroscopy & $5.4 \%$ & $3(0.2)$ & $56(0.7)$ & \\
\hline miscellaneous & $12.5 \%$ & $5(0.4)$ & $35(0.4)$ & \\
\hline Kidney & $21.7 \%$ & $831(58.8)$ & $3,005(38.8)$ & $<0.001$ \\
\hline Partial nephrectomy & $20.0 \%$ & $278(19.7)$ & $1,110(14.2)$ & \\
\hline Unilateral radical nephrectomy & $24.9 \%$ & $525(37.1)$ & $1,583(20.3)$ & \\
\hline Bilateral nephrectomy & $0 \%$ & $0(0.0)$ & $1(0.0)$ & \\
\hline Nephrolithotomy & $8.8 \%$ & $27(1.9)$ & $280(3.6)$ & \\
\hline Pyeloplasty & $3.1 \%$ & $1(0.1)$ & $31(0.4)$ & \\
\hline
\end{tabular}

TUR-B, transurethral bladder resection, TUR-P, transurethral prostate resection, HoLEP, Holmium Laser Enucleation of the prostate

Included numbers of surgery cases were presented as $n(\%)$.

ancidence of postoperative hyponatremia in each subtypes of surgery (in each row) was shown.

of ESRD regarding postoperative $\mathrm{Na}$ levels, an increased risk of progression to ESRD was observed with lower levels of postoperative serum $\mathrm{Na}$ (Figure 3). Moreover, after adjustment with multiple risk factors, which were revealed to be significant in the above analyses, development of postoperative hyponatremia was an independent risk factor associated with progression to ESRD (adjusted HR 1.343, 95\% CI 1.082-1.680, $P=0.007$ ).

\section{Long-term renal outcomes of postoperative hyponatremia}

During a median follow-up duration of $2.6(1.0-4.9)$ years in the study cohort, there were 1,366 secondary composite outcomes, which was the long-term renal prognosis outcome consisting of the composition of progression to ESRD and doubling of $\mathrm{sCr}$ or halving 
of eGFR from baseline. Among them, patients with postoperative hyponatremia showed worse secondary outcomes than the others (Figure 4). Our multivariable analysis revealed that development of postoperative hyponatremia remained a significant risk factor (adjusted HR 1.331, 95\% CI 1.148-1.542, $P<0.001$ ) for worse renal prognosis along with other well-known risk factors, such as old age, male sex, preoperative anemia, preoperative or postoperative kidney function measured by serum creatinine level, and history of diabetes mellitus (Table 6).

Considering the heterogeneity of our study population, we further divided our study cohort according to clinically important characteristics and assessed the effect of new-onset postoperative hyponatremia in each subpopulation (Table 7). The association between postoperative hyponatremia and worse renal outcome was greater in younger, male patients. The association was significant regardless of preexisting reduced kidney function (eGFR $<60 \mathrm{~mL} /$ $\mathrm{min} / 1.73 \mathrm{~m}^{2}$ ). Postoperative hyponatremia for prostate (adjusted HR 1.729, 95\% CI 1.145-2.612, $P=0.009$ ) and kidney (adjusted HR 1.339, 95\% CI 1.099-1.632, $P=0.004$ ) surgery patients was significantly associated with a worse secondary outcome.

\section{DISCUSSION}

In this study, we examined the incidence, risk factors, and outcomes of new-onset postoperative hyponatremia in urologic surgery. Hyponatremia was a common electrolyte disorder after major urologic operations, especially in patients with high-risk perioperative characteristics. Decreased sodium level was associated with increased risk of progression to ESRD. A worse composite renal outcome was related to the development of postoperative hyponatremia, and the results remained significant even after adjustment for multiple clinical factors.

Previous studies showed that postoperative hyponatremia was largely a phenomenon caused by surgical stress, and that pathophysiologic changes in antidiuretic hormonal release play an important role in water retention and decreased serum $\mathrm{Na}$ [16]. In a similar vein, patients with comorbidities and other risk factors were considered to be vulnerable to such stress during operation, and, therefore, to the development of postoperative hyponatremia. Our study results demonstrate that high-risk perioperative status was closely related to the development of postoperative hyponatremia, agreeing with previous studies. Predominant use of normal saline,

ESRD progression

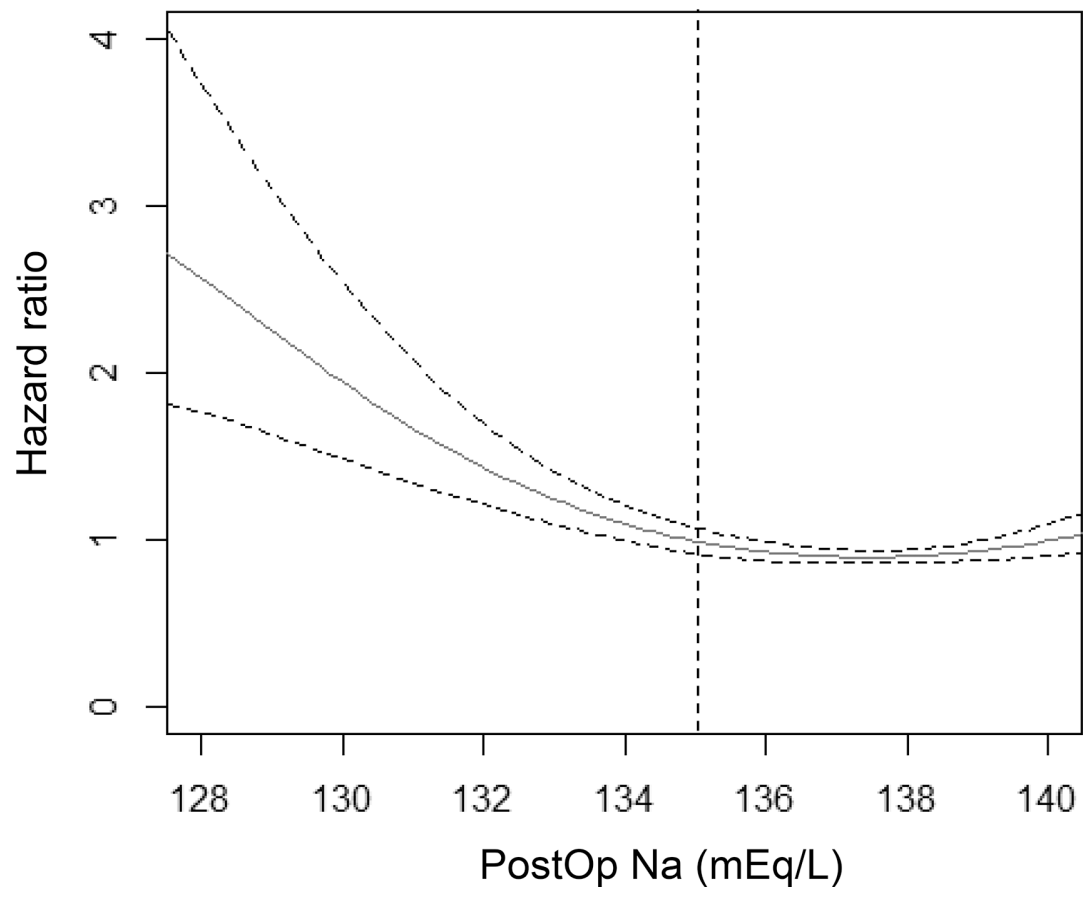

Figure 3: Cox proportional hazard models with panelized smoothing splines showing the association of postoperative Na level and risk of progression to ESRD. The hazard ratio was calculated using the Na level with lowest risk for ESRD as the reference value. The $\mathrm{X}$-axis shows the lowest postoperative sodium level within 7 days from surgery and the Y-axis shows the hazard ratios. Broken lines signify $95 \%$ confidence interval. The vertical broken line is drawn at a sodium level of $135 \mathrm{mEq} / \mathrm{L}$, which was the criterion used to define postoperative hyponatremia in the study. 


\begin{tabular}{|c|c|c|c|}
\hline & $\begin{array}{c}\text { New-onset } \\
\text { hyponatremia }(+) \\
(N=1,414)\end{array}$ & $\begin{array}{c}\text { New-onset } \\
\text { hyponatremia }(-) \\
(N=7,792)\end{array}$ & $P$ value \\
\hline Surgery type & & & 0.906 \\
\hline Open surgery & $713(50.4)$ & $3936(50.5)$ & \\
\hline Laparoscopic surgery & $183(12.9)$ & $1006(12.9)$ & \\
\hline Endoscopic surgery & $335(23.7)$ & $1891(24.3)$ & \\
\hline Robot-assisted surgery & $183(12.9)$ & $959(12.3)$ & \\
\hline Anesthesia type & & & 0.067 \\
\hline Spinal & $117(8.4)$ & $819(10.6)$ & \\
\hline General & $1,267(91.0)$ & $6,791(88.3)$ & \\
\hline Others (MAC, epidural, etc.) & $8(0.6)$ & $80(1.1)$ & \\
\hline ASA classification & & & 0.002 \\
\hline 0 & $4(0.3)$ & $29(0.4)$ & \\
\hline 1 & $455(33.3)$ & $2,629(34.7)$ & \\
\hline 2 & $793(58.0)$ & $4,470(59.1)$ & \\
\hline 3 & $112(8.2)$ & $434(5.7)$ & \\
\hline$\geq 4$ & $3(0.2)$ & $4(0.1)$ & \\
\hline NYHA classification & & & 0.092 \\
\hline 1 & $1,182(89.5)$ & $6,683(91.2)$ & \\
\hline 2 & $117(8.9)$ & $574(7.8)$ & \\
\hline$\geq 3$ & $22(1.7)$ & $73(1.0)$ & \\
\hline Anesthesia time (minutes) & $190(145-250)$ & $175(115-235)$ & $<0.001$ \\
\hline$<120$ & $194(14.0)$ & $1994(26.0)$ & \\
\hline$\geq 120$, and $<180$ & $381(27.4)$ & $1894(24.7)$ & \\
\hline$\geq 180$ & $813(58.6)$ & $3777(49.3)$ & \\
\hline In non-general anesthesia (minutes) & $145(85-170)$ & $115(60-160)$ & $<0.001$ \\
\hline In general anesthesia (minutes) & $200(150-255)$ & $185(125-245)$ & $<0.001$ \\
\hline Surgical time (minutes) & $147(109-200)$ & $135(77-190)$ & $<0.001$ \\
\hline$<120$ & $438(31.6)$ & $3176(41.5)$ & \\
\hline$\geq 120$, and $<180$ & $466(33.6)$ & $2264(29.5)$ & \\
\hline$\geq 180$ & $483(34.8)$ & $2222(29.0)$ & \\
\hline In non-general anesthesia (minutes) & $109(50-135)$ & $85(35-125)$ & 0.001 \\
\hline In general anesthesia (minutes) & $155(110-209)$ & $140(87-197)$ & $<0.001$ \\
\hline Emergency operation & $3(0.2)$ & $25(0.3)$ & 0.505 \\
\hline \multicolumn{4}{|l|}{ Intraoperative use of intravenous fluid } \\
\hline \multicolumn{4}{|l|}{ Balanced salt solution } \\
\hline Number of cases & $374(26.4)$ & $2,065(26.5)$ & 0.968 \\
\hline Amount of fluid use (mL) & $800(500-1,200)$ & $800(500-1,150)$ & 0.863 \\
\hline \multicolumn{4}{|l|}{ Hartman solution } \\
\hline Number of cases & $1,175(83.1)$ & $6,244(80.1)$ & 0.010 \\
\hline Amount of fluid use (mL) & $850(600-1,300)$ & $800(500-1,200)$ & $<0.001$ \\
\hline \multicolumn{4}{|l|}{ Normal saline } \\
\hline Number of cases & $531(37.6)$ & $2,357(30.2)$ & $<0.001$ \\
\hline
\end{tabular}


Amount of fluid use $(\mathrm{mL})$

Hydroxyethyl starch

Number of cases

Amount of fluid use (mL)

$500(500-825)$

Intraoperative RBC transfusion

1-2 pack

$114(8.1)$

3-4 pack

5-9 pack

$>9$ pack

$18(1.3)$

Inotropic agents use at Op day

$700(400-1,000)$

0.136

MAC, monitored anesthesia care, RBC, red blood cell, Op, operation

Values were presented as $n(\%)$ for categorical variables, and median (25\% to $75 \%$ interquartile) for continuous variables (all data in the table showed non-normal distribution).

Hartman solution, and hydroxyethyl starch in the study group might also have been related to hemodynamic instability during surgery, as these fluids are commonly used for intraoperative volume expansion. Longer surgical time was also significantly associated with the development of electrolyte disturbance, as the patients might have experienced more hemodynamic changes during the perioperative period. Additionally, women are known to be prone to develop postoperative hyponatremia due to differences in hormone response and smaller total distribution volume; our findings correspond with this knowledge $[5,6]$. Moreover, many known risk factors, such as older age and chronic comorbidities, were also confirmed to be independently associated with new-onset postoperative hyponatremia in urologic surgery $[3,5,6,9]$.

Furthermore, we demonstrated that postoperative hyponatremia was closely related to poor prognosis in regards to progression to ESRD, and composition of adverse renal outcomes. Considering that the electrolyte imbalance was an independent predictor for worse prognosis, even after adjustment for multiple risk factors, clinicians should closely monitor possible kidney function deterioration in patients with postoperative hyponatremia. In addition, pre- and postoperative $\mathrm{sCr}$ levels or AKI might have been the most commonly considered predictor for renal outcome, but the association of postoperative hyponatremia with poor renal prognosis remained significant, even after adjusting for $\mathrm{sCr}$ values. The reasons for the above results include: 1) as mentioned above, postoperative hyponatremia is related to stressful conditions during surgery [16], implying that patients with an electrolyte imbalance would suffer from more postoperative complications and have a worse clinical prognosis; 2) as patients with postoperative hyponatremia had more comorbidities, the electrolyte imbalance might be an

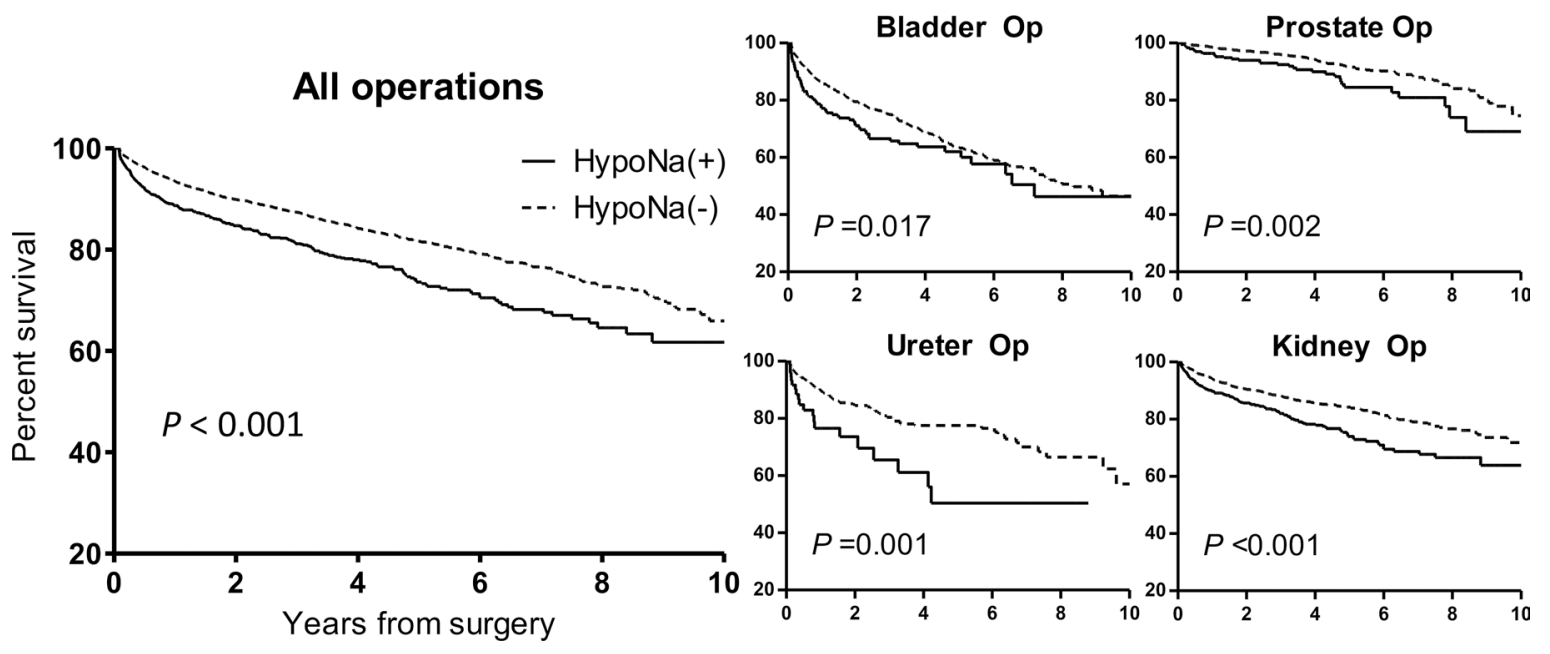

Figure 4: Kaplan-Meier survival curves of the composite renal outcome in all operations and each category of surgery, according to the presence of new-onset postoperative hyponatremia. The composite renal outcome consisted of events of progression to ESRD, serum creatinine doubling or estimated glomerular filtration rate halving form baseline. The X-axis shows the duration from the surgery by years, and the Y-axis shows the percent survival of the composite renal outcome. HypoNa (+) indicates the study group with postoperative hyponatremia, and HypoNa (-) indicates the control group without hyponatremia. The solid line represents the survival curve of the study group and the fine dotted line shows the survival curve of the control group. 


\begin{tabular}{|c|c|c|c|}
\hline & $\begin{array}{c}\text { New-onset } \\
\text { hyponatremia }(+) \\
(N=1,414)\end{array}$ & $\begin{array}{c}\text { New-onset } \\
\text { hyponatremia }(-) \\
(N=7,792)\end{array}$ & $P$ value \\
\hline Acute kidney injury & & & $<0.001$ \\
\hline Stage 1 & $415(30.3)$ & $1,410(20.9)$ & \\
\hline Stage 2 & $26(1.9)$ & $78(1.2)$ & \\
\hline Stage 3 & $49(3.6)$ & $151(2.2)$ & \\
\hline $\mathrm{sCr}(\mathrm{mg} / \mathrm{dL})$ & $1.10(0.91-1.40)$ & $1.03(0.90-1.28)$ & $<0.001$ \\
\hline eGFR $\left(\mathrm{mL} / \mathrm{min} / 1.73 \mathrm{~m}^{2}\right)$ & $55.0(40.0-74.6)$ & $59.0(45.5-74.9)$ & $<0.001$ \\
\hline Sodium (mEq/L) & $133(132-134)$ & $137(136-139)$ & $<0.001$ \\
\hline Hemoglobin (g/dL) & $10.9(9.6-12.0)$ & $11.4(10.2-12.6)$ & $<0.001$ \\
\hline Sodium decrement $(\mathrm{mEq} / \mathrm{L})$ & $7.0(9.0-5.0)$ & $3.0(5.0-2.0)$ & $<0.001$ \\
\hline Hemoglobin decrement (g/dL) & $2.2(1.1-3.3)$ & $2.1(1.1-3.2)$ & 0.038 \\
\hline Body weight change (kg) & $0.0(-1.0-1.0)$ & $0.0(-1.0-1.0)$ & 0.511 \\
\hline Days of hospital stay (days) & $6(5-9)$ & $5(4-8)$ & $<0.001$ \\
\hline Postoperative ICU admission & $14(1.0)$ & $48(0.6)$ & 0.114 \\
\hline
\end{tabular}

sCr, serum creatinine, eGFR, estimated glomerular filtration rate, ICU, intensive care unit.

Values were presented as $n(\%)$ for categorical variables, and median ( $25 \%$ to $75 \%$ interquartile) for continuous variables (all data in the table showed non-normal distribution).

indicator of severe illness; however, our multivariable analysis suggested that new-onset postoperative hyponatremia was an independent risk factor, even from pre- and post-operative creatinine levels, for worse renal outcome; 3) patients with postoperative hyponatremia had longer hospital stays, so exposure to additional inhospital complications such as infection could have been attributed to adverse outcomes [3, 4, 9]; and 4) although rare, direct complications of hyponatremia, such as neurologic symptoms, might also have contributed to a worse prognosis $[17,18]$.

The association between the composite renal prognosis and new-onset postoperative hyponatremia differed among subpopulations in our study. Although young, male patients were less prone to experience postoperative hyponatremia, when developed, the clinical significance of the electrolyte imbalance was greater in these patients. Because patients with postoperative hyponatremia who underwent prostate or kidney operation had a worse renal prognosis, clinicians should pay careful attention to the electrolyte imbalance in these patients. Particularly, as preserving kidney function is a critical problem for patients who undergo nephrectomy, which was the most common kidney surgery in this study cohort, not only the pre- and post-operative serum creatinine levels, but also the electrolyte imbalance after surgery should be considered as predictive of poor renal prognosis. Moreover, although technical advances have decreased the incidence of TUR-P syndrome [10], postoperative hyponatremia remains an important risk factor for worse renal outcome in prostate operations. The types of bladder and ureter operations included in our study were relatively minor surgeries compared to the others, and renal deterioration occurred less in these patients, which may be why the association between hyponatremia development and worse composite renal outcome was not prominent in these patients.

Several limitations should be noted in our study. First, as this was a retrospective, observational study, our study cohort did not receive standardized treatment or follow up after their operations. In addition, detailed surgical category and postoperative fluid administration strategies were heterogeneous. However, the study design enabled us to evaluate the clinical significance of postoperative hyponatremia in a large cohort of urologic surgery cases with sufficient statistical power. Second, minor surgeries without follow up samples of serum electrolytes were excluded as were some operation categories. Hence, our findings may not be applicable to these other surgery categories. Third, the exact mechanism for worsened prognosis after postoperative hyponatremia could not be determined by our study results. Lastly, as this study was a bi-center study in a single nation, the study results may not be generalizable to patients of other centers with a different medical environment.

In conclusion, postoperative hyponatremia in urologic surgery was common and was related to high-risk perioperative characteristics. Considering that the presence of postoperative hyponatremia was strongly associated with consequent worse renal prognosis, clinicians should carefully consider such adverse outcomes in patients with this electrolyte imbalance, and evaluate the cause of hyponatremia and coexisting comorbidities in these patients. 
Table 5: Risk factors for new-onset postoperative hyponatremia

\begin{tabular}{|c|c|c|}
\hline Variables & ${ }^{\mathrm{b}}$ Adjusted OR (95\% CI) & $P$ value \\
\hline Age (1-year increment) & $1.013(1.007-1.018)$ & $<0.001$ \\
\hline Female sex (vs. male) & $1.216(1.045-1.414)$ & 0.011 \\
\hline Body mass index ( $1 \mathrm{~kg} / \mathrm{m}^{2}$ increment $)$ & $0.999(0.999-1.000)$ & $<0.001$ \\
\hline Cancer (vs. no) & $1.248(1.067-1.459)$ & 0.006 \\
\hline Hypertension (vs. no) & $1.139(0.995-1.305)$ & 0.060 \\
\hline Diabetes mellitus (vs. no) & $1.317(1.116-1.554)$ & 0.001 \\
\hline Baseline $\mathrm{Na}(1 \mathrm{mEq} / \mathrm{L}$ increment $)$ & $0.829(0.803-0.856)$ & $<0.001$ \\
\hline Baseline anemia (hemoglobin $<12 \mathrm{~g} / \mathrm{dL}$ ) & $1.311(1.139-1.509)$ & $<0.001$ \\
\hline Kidney operation (vs. other surgery) & $2.765(2.388-3.202)$ & $<0.001$ \\
\hline Surgery time (1-hour increment) & $1.109(1.063-1.157)$ & $<0.001$ \\
\hline NYHA classification $(\geq 2)$ & $1.159(0.905-1.485)$ & 0.243 \\
\hline ASA classification ( $\geq$ class III) & $1.621(1.372-1.915)$ & $<0.001$ \\
\hline Use of Hartman solution (vs. no) & $1.319(1.107-1.573)$ & 0.002 \\
\hline Use of hydroxyethyl starch (vs. no) & $1.139(0.978-1.325)$ & 0.094 \\
\hline Use of normal saline (vs. no) & $1.236(1.079-1.416)$ & 0.002 \\
\hline Transfusion amount of RBC (1-pack increment) & $1.021(0.984-1.060)$ & 0.274 \\
\hline \multicolumn{3}{|l|}{ Acute kidney injury (vs. no acute kidney injury) } \\
\hline Stage 1 & $1.433(1.232-1.666)$ & $<0.001$ \\
\hline Stage 2 & $1.649(1.023-2.657)$ & 0.040 \\
\hline Stage 3 & $1.724(1.214-2.447)$ & 0.002 \\
\hline
\end{tabular}

OR, odds ratio, $\mathrm{CI}$, confidence interval

${ }^{\mathrm{b}}$ Adjusted simultaneously with all variables in the table and multivariable logistic regression test was performed.

Table 6: Clinical characteristics related to long-term renal prognosis in the study cohort

\begin{tabular}{lccc}
\hline Variables & ${ }^{a}$ Adjusted HR & $\mathbf{9 5 \%}$ CI & $P$ value \\
\hline Age (years) & 1.033 & $1.027-1.039$ & $<0.001$ \\
Female sex & 0.700 & $0.593-0.827$ & $<0.001$ \\
Hypertension & 1.025 & $0.897-1.172$ & 0.713 \\
Diabetes mellitus & 1.427 & $1.216-1.675$ & $<0.001$ \\
Preoperative severe anemia & 1.282 & $1.036-1.586$ & 0.022 \\
(Hb $<8.0 \mathrm{~g} / \mathrm{dL})$ & & & $<0.001$ \\
Preoperative creatinine (mg/dL) & 2.022 & $1.711-2.389$ & 0.003 \\
Postoperative creatinine (mg/dL) & 1.010 & $1.004-1.017$ & 0.656 \\
Intraoperative use of normal saline & 1.030 & $0.905-1.172$ & $<0.001$ \\
Postoperative hyponatremia & 1.331 & $1.148-1.542$ & \\
\hline
\end{tabular}

HR, hazard ratio, CI, confidence interval, hb, hemoglobin

${ }^{a}$ Adjusted with organ of surgery (bladder, prostate, ureter, kidney), surgery type (open, laparoscopic, endoscopic, and robotassisted surgery), age, sex, history of hypertension, diabetes mellitus, preoperative severe anemia, intraoperative use of normal saline, preoperative and postoperative serum creatinine level. 
Table 7: Association of postoperative hyponatremia and long-term renal prognosis in various subgroups

\begin{tabular}{lccc}
\hline Subpopulation & ${ }^{a}$ Adjusted HR & $\mathbf{9 5 \% ~ C I ~}$ & P value \\
\hline Age $\geq 70(N=3,060)$ & 1.088 & $0.861-1.376$ & 0.479 \\
Age $50-70(N=4,670)$ & 1.490 & $1.205-1.843$ & $<0.001$ \\
Age $<50(N=1,476)$ & 1.918 & $1.245-2.957$ & 0.003 \\
\hline Female $(N=2,032)$ & 1.227 & $0.873-1.725$ & 0.239 \\
Male $(N=7,174)$ & 1.362 & $1.156-1.605$ & $<0.001$ \\
\hline eGFR $<60(N=3,232)$ & 1.299 & $1.066-1.584$ & 0.010 \\
eGFR $\geq 60(N=5,974)$ & 1.425 & $1.142-1.778$ & 0.002 \\
\hline Bladder operation $(N=1,814)$ & 1.118 & $0.830-1.508$ & 0.463 \\
Prostate operation $(N=2,957)$ & 1.729 & $1.145-2.612$ & 0.009 \\
Ureter operation $(N=599)$ & 1.634 & $0.899-2.971$ & 0.107 \\
Kidney operation $(N=3,836)$ & 1.339 & $1.099-1.632$ & 0.004 \\
\hline
\end{tabular}

HR, hazard ratio, CI, confidence interval, eGFR, estimated glomerular filtration rate $\left(\mathrm{mL} / \mathrm{min} / 1.73 \mathrm{~m}^{2}\right)$

${ }^{a}$ Adjusted with organ of surgery (bladder, prostate, ureter, kidney), surgery type (open, laparoscopic, endoscopic, and robotassisted surgery), age, sex, history of hypertension, diabetes mellitus, preoperative severe anemia, intraoperative use of normal saline, preoperative and postoperative serum creatinine level.

\section{MATERIALS AND METHODS}

\section{Ethics statement}

This study was approved by the institutional review boards of Seoul National University Hospital (J-1407145-597) and Seoul National University Boramae Medical Center (16-2014-96). This study was conducted following the principles of the Declaration of Helsinki. As the study was a retrospective, observational study without medical intervention, informed consent was waived.

\section{Study cohort}

The study was a retrospective cohort study including patients from two tertiary hospitals in Korea. The inclusion criteria were bladder, prostate, ureter, and kidney surgery cases performed in the urologic department, with patients aged $\geq 18$ years and available postoperative follow-up serum $\mathrm{Na}$ level within 7 days. The following patients were excluded from the study: 1) those with preexisting ESRD; 2) with no preoperative sCr within 1 month; 3) with no documented surgical record; 4) other urology surgeries than the four major categories identified in this study or complex operation cases involving multiple organs, 5) those lost to follow up less than 1 month post-operation due to causes other than death or the start of RRT, and 6) patients who had baseline hyponatremia. The study group consisted of patients with newly developed postoperative hyponatremia, defined as at least one serum sodium level lower than $135 \mathrm{mEq} / \mathrm{L}$ within 7 days after surgery, and the others were included in the control group.

\section{Data collection}

We collected the following preoperative demographic, laboratory, and clinical information of study subjects by EHR review: age; sex; weight; height; body mass index; baseline and follow-up levels of serum sodium and potassium; all measured values of $\mathrm{sCr}$ in each patient and eGFR values, calculated using the Chronic Kidney Disease Epidemiology Collaboration (CKDEPI) equation [19], and baseline hemoglobin levels. Comorbidity, including cancer, hypertension, and diabetes, were collected by reviewing the designated International Classification of Diseases-10 (ICD-10) diagnostic codes and medical records using relevant medications. Liver cirrhosis, or heart failure requiring admission, was also documented by reviewing the admission records of the patients. Presence of baseline chronic kidney disease was defined by a baseline eGFR less than $60 \mathrm{ml} / \mathrm{min} / 1.73$ $\mathrm{m}^{2}$, which isa common definition for stage 3 CKD [24]. Preoperative severe anemia was defined by a hemoglobin level less than $8 \mathrm{~g} / \mathrm{dL}$ at baseline. The last laboratory values within 1 month before operation were regarded as the baseline levels.

Considering that the association between medication use and hyponatremia was well-reviewed [20], we collected information on the following medication use: ACE inhibitor or ARB, diuretics, NSAIDs, proton pump inhibitor, antiepileptic medication, thyroidal hormone replacement, antithyroidal medications (prophylthiouracil and methimazol), oral steroid, and serononin-norepinephrine reuptake inhibitor/selective serotonin reuptake inhibitor. 
The following perioperative and intraoperative characteristics were reviewed: operation type, whether the surgery was an emergency operation, anesthesia type, risk stratification score including ASA classification [21] and NYHA classification [22] recorded by an anesthesiologist, total anesthesia time, total surgical time, type and amount of intraoperative administered fluids, intraoperative transfusion amount of $\mathrm{RBC}$, and perioperative use of intravenous inotropic agents.

In addition, postoperative laboratory values and body weight changes within 7 days after the operation were collected. Postoperative AKI was staged by KDIGO clinical practice guideline for AKI [23], and also defined with $\mathrm{sCr}$ escalation within 7 days after operation. Information regarding postoperative intensive care unit admission and days of hospital stay wasalso documented.

\section{Outcome measurement}

The primary renal outcome was defined as the event of progression to ESRD in the postoperative period. Progression to ESRD was defined by the start of maintenance dialysis or new progression to CKD stage 5 $\left(\right.$ GGFR $\left.<15 \mathrm{ml} / \mathrm{min} / 1.73 \mathrm{~m}^{2}\right)$ [24]. The secondary outcome was a combination of additional long-term renal outcomes. The secondary outcome was defined as a composition of progression to ESRD, and $\mathrm{sCr}$ doubling/eGFR halving from baseline. All outcomes were defined and collected before the start of the analysis.

\section{Statistical analysis}

Data are presented as frequencies and percentages for categorical variables and were analyzed by chisquared tests. Continuous variables were expressed as the mean (standard deviation) or median scores (interquartile ranges) depending on the results of ShapiroWilk normality test. To assess risk factors for new-onset postoperative hyponatremia, we performed multivariable logistic regression with adjustment for variables that were regarded as clinically relevant and showed a significant difference between the study and the control groups. The renal outcomes were evaluated using the Cox regression hazard model. The association between postoperative sodium level and risk of the primary outcome was investigated by penalized spline analysis [25]. Multivariable analyses were adjusted per the following variables: age, sex, history of hypertension, diabetes mellitus, preoperative severe anemia, intraoperative use of normal saline, preoperative and postoperative serum creatinine level, main organ of surgery (bladder, prostate, ureter, or kidney), and surgery type (open, laparoscopic, endoscopic or robot-assisted). We used a complete case analysis method, as there were 253 cases of missing preoperative hemoglobin levels; no missing information was identified in other variables included in the multivariable analysis. All statistical analyses were performed using R package version 3.4.0 (R Development Core Team, Austria). Two-sided $p$ values with a statistical significance level of 0.05 were used.

\section{Abbreviations}

AKI, acute kidney injury; RBC, red blood cell; TUR-P, transurethral resection of prostate; eGFR, estimated glomerular filtration rate; CKD-EPI, Chronic Kidney Disease Epidemiology Collaboration; ACE $\mathrm{I}$, angiotensin converting enzyme; ARB, angiotensin receptor blocker; NSAID, non-steroidal anti-inflammatory drugs; Na, sodium; OR, odds ratio; HR, hazard ratio; CI, confidence interval; sCr, serum creatinine; ESRD, end stage renal disease.

\section{Author contributions}

The research idea was conceived by SP, JNA, and CSL. JPL, YKO, KWJ, and CSL participated in the study design formulation. SP wrote the original manuscript. SP, JNA, JPL, DKK, KWJ, and YSK participated in the data collection. YKO, DKK, KWJ, YSK, and CSL helped in the manuscript correction and provided statistical knowledge. CSL supervised the overall study and approved the final version of the manuscript.

\section{ACKNOWLEDGMENTS}

None.

\section{CONFLICTS OF INTEREST}

None.

\section{FUNDING}

None.

\section{REFERENCES}

1. Upadhyay A, Jaber BL, Madias NE. Incidence and prevalence of hyponatremia. Am J Med. 2006; 119:S30-35.

2. Wald R, Jaber BL, Price LL, Upadhyay A, Madias NE. Impact of hospital-associated hyponatremia on selected outcomes. Arch Intern Med. 2010; 170:294-302.

3. Hennrikus E, Ou G, Kinney B, Lehman E, Grunfeld R, Wieler J, Damluji A, Davis C 3rd, Mets B. Prevalence, Timing, Causes, and Outcomes of Hyponatremia in Hospitalized Orthopaedic Surgery Patients. J Bone Joint Surg Am. 2015; 97:1824-1832.

4. Rudge JE, Kim D. New-onset hyponatraemia after surgery for traumatic hip fracture. Age Ageing. 2014; 43:821-826. 
5. Hussain NS, Piper M, Ludlam WG, Ludlam WH, Fuller CJ, Mayberg MR. Delayed postoperative hyponatremia after transsphenoidal surgery: prevalence and associated factors. J Neurosurg. 2013; 119:1453-1460.

6. Mohan S, Gu S, Parikh A, Radhakrishnan J. Prevalence of hyponatremia and association with mortality: results from NHANES. Am J Med. 2013; 126:1127-37.e1.

7. Nakahira J, Sawai T, Fujiwara A, Minami T. Transurethral resection syndrome in elderly patients: a retrospective observational study. BMC Anesthesiol. 2014; 14:30.

8. Issa MM, Young MR, Bullock AR, Bouet R, Petros JA. Dilutional hyponatremia of TURP syndrome: a historical event in the 21 st century. Urology. 2004; 64:298-301.

9. Antonelli Incalzi R, Gemma A, Capparella O, Terranova L, Sanguinetti C, Carbonin PU. Post-operative electrolyte imbalance: its incidence and prognostic implications for elderly orthopaedic patients. Age Ageing. 1993; 22:325-331.

10. Issa MM. Technological advances in transurethral resection of the prostate: bipolar versus monopolar TURP. J Endourol. 2008; 22:1587-1595.

11. Kim CS, Bae EH, Ma SK, Kweon SS, Kim SW. Impact of partial nephrectomy on kidney function in patients with renal cell carcinoma. BMC Nephrol. 2014; 15:181.

12. Cho A, Lee JE, Kwon GY, Huh W, Lee HM, Kim YG, Kim DJ, Oh HY, Choi HY. Post-operative acute kidney injury in patients with renal cell carcinoma is a potent risk factor for new-onset chronic kidney disease after radical nephrectomy. Nephrol Dial Transplant. 2011; 26:3496-3501.

13. Kwon T, Jeong IG, Lee C, You D, Hong B, Hong JH, Ahn H, Kim CS. Acute Kidney Injury After Radical Cystectomy for Bladder Cancer is Associated with Chronic Kidney Disease and Mortality. Ann Surg Oncol. 2016; 23:686-693.

14. Rajan S, Babazade R, Govindarajan SR, Pal R, You J, Mascha EJ, Khanna A, Yang M, Marcano FD, Singh AK, Kaouk J, Turan A. Perioperative factors associated with acute kidney injury after partial nephrectomy. Br J Anaesth. 2016; 116:70-76.

15. Kheterpal S, Tremper KK, Englesbe MJ, O'Reilly M, Shanks AM, Fetterman DM, Rosenberg AL, Swartz RD. Predictors of postoperative acute renal failure after noncardiac surgery in patients with previously normal renal function. Anesthesiology. 2007; 107:892-902.

16. Steele A, Gowrishankar M, Abrahamson S, Mazer CD, Feldman RD, Halperin ML. Postoperative hyponatremia despite near-isotonic saline infusion: a phenomenon of desalination. Ann Intern Med. 1997; 126:20-25.

17. Fujisawa $H$, Sugimura $Y$, Takagi $H$, Mizoguchi $H$, Takeuchi H, Izumida H, Nakashima K, Ochiai H, Takeuchi S, Kiyota A, Fukumoto K, Iwama S, Takagishi Y, et al. Chronic Hyponatremia Causes Neurologic and Psychologic Impairments. J Am Soc Nephrol. 2016; 27:766-780.

18. Renneboog B, Musch W, Vandemergel X, Manto MU, Decaux G. Mild chronic hyponatremia is associated with falls, unsteadiness, and attention deficits. Am J Med. 2006; 119:71.e1-8.

19. Levey AS, Stevens LA, Schmid CH, Zhang YL, Castro AF 3rd, Feldman HI, Kusek JW, Eggers P, Van Lente F, Greene $\mathrm{T}$, Coresh J. A new equation to estimate glomerular filtration rate. Ann Intern Med. 2009; 150:604-612.

20. Liamis G, Milionis H, Elisaf M. A review of drug-induced hyponatremia. Am J Kidney Dis. 2008; 52:144-153.

21. Dripps RD. New classification of physical status. Anesthesiology. 1963; 24:111.

22. The Criteria Committee of the New York Heart Association. Nomenclature and Criteria for Diagnosis of Diseases of the Heart and Great Vessels. 9th ed. Boston, Mass: Little, Brown \& Co. 1994:253-256.

23. Kidney Disease: Improving Global Outcomes (KDIGO) Work Group. KDIGO clinical practice guideline for acute kidney injury. Kidney Int Suppl. 2012; 2:1-138.

24. Kidney Disease: Improving Global Outcomes (KDIGO) Work Group: KDIGO 2012 Clinical Practice guideline for the evaluation and managementof chronic kidney disease. Kidney Int Suppl. 2013; 3:136-50.

25. Eilers PH, Marx BD. Flexible smoothing with B-splines and penalties. Stat Sci. 1996; 11:89-102. 